\title{
Determinants connected with the Periodic Solutions of Mathieu's Equations.
}

By A. G. Burgess, Research Student, Edinburgh University Mathematical Iaboratory.

(Read 11th June 1915. Received 28th June 1915.)

\section{\$1. Introduction.}

Various solutions of Mathieu's equation, ${ }^{*}$ or the equation of the elliptic cylinder functions

$$
\frac{d^{2} y}{d z^{2}}+(a+16 q \cos 2 z) y=0
$$

have recently been discussed in an elegant series of papers in these Proceedings. $\dagger$ These papers have dealt with the periodic and quasi-periodic solutions, but the present paper merely considers determinants which give the infinite series of relations between $a$ and $q$, so that the solutions are purely periodic, i.e. the solutions denoted by Professor Whittaker $\ddagger$

$$
\begin{array}{rlll}
c e_{0}(z), c e_{1}(z) & \ldots & c e_{n}(z) \\
s e_{1}(z) & \ldots & s e_{n}(z) .
\end{array}
$$

The first set of determinants are derived from Lamés equation, denoted in the Riemann notation by

$$
y=P\left|\begin{array}{cccc}
0 & b^{2} & c^{2} & \infty \\
0 & 0 & 0 & -\frac{n}{2} x \\
\frac{1}{2} & \frac{1}{2} & \frac{1}{2} & \frac{1}{2}+\frac{n}{2}
\end{array}\right|
$$

and the other set by means of G. W. Hill's method.

\footnotetext{
* Liouville's Journal, sér 2, t. xiii., pp. 137-203.

† Proceedings of Edinburgh Mathematical Society, Vols. XXXII. and XXXIII. (Part 1.)

$\ddagger$ Proceedings of the Mathematical Congress, 1912, Vol. I.

§ Hill : Acta Mathematica, Vol. VIII., pp. 1-36, 1886.
} 
Afterwards it is shown how the equations which give one set of determinants can be got from the equations corresponding to the other set.

\section{§2. By Lamés Equation.}

Lamé's equation is

$$
\begin{aligned}
x\left(x-b^{2}\right)\left(x-c^{2}\right) \frac{d^{2} y}{d x^{2}}+\frac{1}{2}\left(3 x^{2}-2 b^{2} x-2 c^{2} x+b^{2} c^{2}\right) \frac{d y}{d x} & \\
& -\frac{1}{4}\{n(n+1) x+A\} y=0 .
\end{aligned}
$$

Divide throughout by $c^{2}$, and make $c \rightarrow \infty$, and also $n$ and $A$, but so that $\frac{n(n+1)}{c^{2}}$ and $\frac{A}{c^{2}}$ remain finite, and the equation becomes

$$
\begin{array}{r}
x\left(x-b^{2}\right) \frac{d^{2} y}{d x^{2}}+\frac{1}{2}\left(2 x-b^{2}\right) \frac{d y}{d x}-(C x+D) y=0 \\
\text { where } C=-\frac{n(n+1)}{c^{2}} \text { and } D=-\frac{A}{4 c^{2}} .
\end{array}
$$

Substituting $b^{2} \cos ^{2} z$ for $x$, we have

$$
\begin{array}{r}
b^{2} \cos ^{2} z\left(b^{2} \cos ^{2} z-b^{2}\right)\left\{\frac{-2 b^{2} \sin z \cos z \frac{d^{2} y}{d z^{2}}+2 b^{2}\left(\cos ^{2} z-\sin ^{2} z\right) \frac{d y}{d z}}{-8 b^{6} \sin ^{3} z \cos ^{3} z}\right\} \\
+\frac{1}{2}\left\{\frac{2 b^{2} \cos ^{2} z-b}{-2 b^{2} \sin z \cos z}\right\} \frac{d y}{d z}-\left(C b^{2} \cos ^{2} z+D\right) y=0 .
\end{array}
$$

This simplifies to $\frac{d^{2} y}{d z^{2}}+\left(4 b^{2} C \cos ^{2} z+4 D\right) y=0$.

Comparing this equation with Mathieu's equation,

$$
\frac{d^{2} y}{d z^{2}}+(a+16 q \cos 2 z) y=0, \text { or } \frac{d^{2} y}{d z^{2}}+\left(a+16 q+32 q \cos ^{2} z\right) y=0,
$$

we see that they are the same equation, if $a-16 q=4 D$ and $32 q=4 b^{2} C$. There is no loss of generality in taking $b^{2}=1$, in which case the equation (1) becomes

$$
\left(-x^{2}+x\right) y^{\prime \prime}+\frac{1}{2}(-2 x+1) y^{\prime}+(C x+D) y=0
$$

where $a-16 q=4 D$ and $32 q=4 C$.

If certain relations exist between $C$ and $D$, the solution of equation (2) can be expressed as a polynomial in $x$, such as 
$y=a_{0}+a_{1} x+a_{2} x^{2} \ldots \quad$ Now, as the substitution was $x=\cos ^{2} z$, the relations between $C$ and $D$ which give the polynomial solutions of equation (2) will give relations under which Mathieu's equation can be solved in the form $y=a_{0}+a_{1} \cos ^{2} z+a_{2} \cos ^{4} z \ldots$ i.e. the $c e_{0}(z), c e_{2}(z), c e_{4}(z)$ solutions, for these can be expressed in terms of $\cos ^{2} z$.

By repeated differentiation of the simplified form of Lame's equation the following series of equations are obtained :-

$$
\begin{aligned}
& \left(-x^{2}+x\right) y^{\prime \prime}+\left(-x+\frac{1}{2}\right) y^{\prime}+(C x+D) y=0 \\
& \left(-x^{2}+x\right) y^{\prime \prime \prime}+\left(-3 x+\frac{3}{2}\right) y^{\prime \prime}+(C x+D-1) y^{\prime}+C y=0 \\
& \left(-x^{2}+x\right) y^{\mathrm{iv}}+\left(-5 x+\frac{5}{2}\right) y^{\prime \prime \prime}+(C x+D-4) y^{\prime \prime}+2 C y^{\prime}=0 \\
& \left(-x^{2}+x\right) y^{\mathrm{v}}+\left(-7 x+\frac{7}{2}\right) y^{\mathrm{iv}}+(C x+D-9) y^{\prime \prime \prime}+3 C y^{\prime \prime}=0
\end{aligned}
$$

etc.

In these equations substitute $x=0, C=8 q, D=\frac{a-16 q}{4}$, and eliminate $y, y^{\prime}, y^{\prime \prime} \ldots$, and the following infinite determinant is

\begin{tabular}{|c|c|c|c|c|}
\hline$a-16 q$ & 2 & 0 & 0 & $0 \ldots \ldots \ldots$ \\
\hline $32 q$ & $a-16 q-2^{2}$ & 6 & 0 & $0 \ldots \ldots$. \\
\hline 0 & $64 q$ & $a-16 q-4$ & 10 & $0 \ldots \ldots \ldots$ \\
\hline 0 & 0 & $96 q$ & $a-16 q-6$ & $14 \ldots \ldots \ldots$ \\
\hline 0 & 0 & 0 & $128 q$ & $a-16 q-8^{2} \ldots$ \\
\hline
\end{tabular}
derived.

$\left|\begin{array}{ccccc}\frac{a-16 q}{4} & \frac{1}{2} & 0 & 0 & 0 \ldots \ldots \ldots \\ 8 q & \frac{a-16 q}{4}-1^{2} & \frac{3}{2} & 0 & 0 \ldots \ldots \ldots \\ 0 & 16 q & \frac{a-16 q}{4}-2^{2} & \frac{5}{2} & 0 \ldots \ldots \ldots \\ 0 & 0 & 24 q & \frac{a-16 q}{4}-3^{2} & \frac{7}{2} \ldots \ldots \ldots \\ 0 & 0 & 0 & 32 q & \frac{a-16 q}{4}-4^{2} \ldots \\ \ldots \ldots \ldots \ldots \ldots \ldots \ldots \ldots \ldots \ldots \ldots \ldots \ldots \ldots \ldots \ldots \ldots \ldots \ldots \ldots \ldots \ldots \ldots \ldots \ldots \ldots \ldots \ldots \ldots \ldots \ldots \ldots \ldots \ldots \ldots \ldots \ldots \ldots \ldots\end{array}\right|=0$

If each row be multiplied by 4 , the determinant becomes 
The leading diagonal shows that when $q=0, a=0,2^{2}, 4^{2}, 6^{2} \ldots$, which is what was to be expected, as the relations between $a$ and $q$ corresponding to the solutions $c e_{0}(z), c e_{2}(z), c e_{4}(z) \ldots$ reduce to these when $q=0$.

The value of the determinant

$$
\left|\begin{array}{ccccc}
a_{1} & b_{1} & 0 & 0 & \ldots \\
c_{2} & a_{2} & b_{2} & 0 & \cdots \\
0 & c_{3} & a_{3} & b_{3} & \ldots \\
0 & 0 & c_{4} & a_{4} & \cdots
\end{array}\right|=\left|\begin{array}{lllll}
a_{1} & 1 & 0 & 0 & \ldots \\
d_{2} & a_{2} & 1 & 0 & \ldots \\
0 & d_{3} & a_{3} & 1 & \ldots \\
0 & 0 & d_{4} & a_{4} & \ldots
\end{array}\right|,
$$

in which the leading diagonals are the same and one of the side diagonals is $1,1,1 \ldots$ provided $d_{r+1}=b_{r} c_{r+1}$.

Accordingly the determinant is equivalent to the continuant

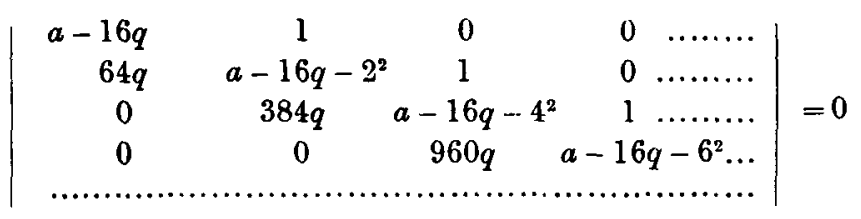

I have adopted the notation $C^{\prime} e l$, Soh' for the determinants derived, so that they may be easily referred to, $c$ and $s$ signifying the determinants corresponding to the $c e(z)$ and $s e(z)$ functions respectively, $e$ and $o$ the even and odd suffixes of $c e(z)$ and $s e(z)$, and $l$ and $h$ denoting whether they were derived my means of Lamé's equation or Hill's method.

This determinant as it stands is divergent. It can be made convergent by dividing each row by certain factors, but the purpose is to truncate the determinant so as to get approximate relations between $a$ and $q$, and hence the rows could be multiplied by these factors again, and the same determinant derived as would be when $C e l$ is truncated.*

The determinant $\mathrm{Cel}$ was truncated to 8 columns and 8 rows. The method of reversion of series was applied and the relation corresponding to the $c e_{0}(z)$ solution was found to be

$$
a=-32 q^{2}+224 q^{4}-\frac{29696}{9} q^{6}+\ldots \dagger
$$

* This remark applies to all the determinants derived.

† Cf. Young: Proceedings of Edinburgh Mathematical Society, Vol. XXXII. Lindsay Ince: Proceedings of Edinburgh Mathematical Society, Vol. XXXIII, (Part 1.) 
In order to get the relation corresponding to $c e_{2}^{\prime}(z), a+2^{2}$ was substituted for $a$. On truncating to 5 columns and 5 rows

$$
a=\frac{80}{3} q^{2}-\frac{6104}{2} q^{4} \ldots
$$

Similarly by substituting $a+4^{2}, a+6^{2}, \ldots$ for $a$, the relations between $a$ and $q$ corresponding to $c e_{4}(z), c e_{6}(z) \ldots$ can be got.

\section{\$3. Determinant corresponding to $c e_{1}(z), c e_{3}(z)$, etc.}

The foregoing suggests that by a different substitution an equation might be derived from which, by a similar method, the relations between $a$ and $q$ corresponding to $c e_{1}(z), c e_{3}(z) \ldots$ might be got.

$c e_{1}(z), c e_{3}(z), \ldots$ can be expressed in terms of $\cos z$. Accordingly the substitution $x=\cos z$ should give determinants corresponding to all the $c e(z)$ solutions.

If $x=\cos z$ be substituted in Mathieu's equation, it becomes

$$
\left(1-x^{2}\right) y^{\prime \prime}-x y^{\prime}+\left(a-16 q+32 q x^{2}\right) y=0 .
$$

By repeated differentiation and substitution of $x=0$, the following sets of equations are got:-

$$
\begin{array}{ll}
y^{\prime \prime}+(a-16 q) y=0 & y^{\prime \prime \prime}+\left(a-16 q-1^{2}\right) y^{\prime}=0 \\
y^{\mathrm{iv}}+\left(a-16 q-2^{2}\right) y^{\prime \prime}+64 q y=0 & y^{\mathrm{v}}+\left(a-16 q-3^{2}\right) y^{\prime \prime \prime}+192 q y^{\prime}=0 \\
y^{\mathrm{vi}}+\left(a-16 q-4^{2}\right) y^{\mathrm{iv}}+384 q y^{\prime \prime}=0 & y^{\mathrm{vii}}+\left(a-16 q-5^{2}\right) y^{v}+640 q y^{\prime \prime \prime}=0
\end{array}
$$

$c e_{0}(z), c e_{2}(z)$, etc., are functions of even powers of $\cos z$; when $\cos z=0, y^{\prime}, y^{\prime \prime \prime}, y^{v} .$. are each zero, whereas $c e_{1}(z), c e_{3}(z)$, etc., are functions of odd powers of $\cos z$, and $y, y^{\prime \prime}, y^{\mathrm{iv}}, y^{\mathrm{i}}, \ldots$ are each zero on a like substitution. Accordingly the first set of equations give relations corresponding to $c e_{0}(z), c e_{2}(z)$, etc., and the second set corresponding to $c e_{1}(z), c e_{3}(z)$, etc.

The first set gives on elimination the same determinant $\mathrm{Cel}$, and the second the determinant

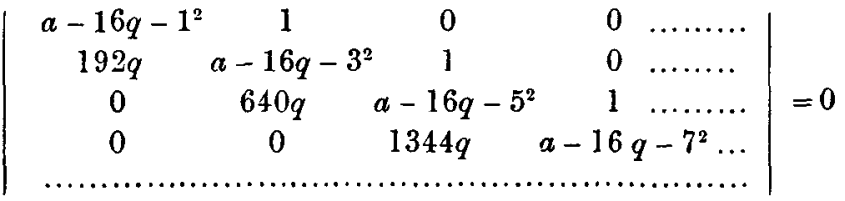


The leading diagonal shows that this corresponds to $c e_{1}(z)$, $c e_{3}(z)$, etc. On substituting $a+1^{2}$ for $a$ and truncating to 4 rows and columns the relation corresponding to $c e_{1}(z)$,

was found.

$$
a=-8 q-8 q^{2}+8 q^{3} \ldots
$$

In like manner the relations for $c e_{3}(z), c e_{5}(z) \ldots$ can be obtained.

$\S 4$. Determinant corresponding to $s e_{1}(z), s e_{3}(z)$, etc.

These are functions of $\sin z$, and, accordingly, the substitution $x=\sin z$ will give an equation from which determinants may be derived corresponding to these solutions and such others as can be expressed as polynomials in $\sin z$, viz. $c e_{0}(z), c e_{2}(z)$, etc.

The substitution of $x=\sin z$ in Mathieu's equation gives

$$
\left(1-x^{2}\right) y^{\prime \prime}-x y^{\prime}+\left(a+16 q-32 q x^{2}\right) y=0 .
$$

Now $c e_{0}(z), c e_{2}(z) \ldots$ can be expressed in terms of even powers of $\sin z$, whereas $s e_{1}(z), s e_{3}(z), \ldots$ only involve the odd powers. Therefore when $\sin z=0($ i.e. $x=0), y^{\prime}, y^{\prime \prime \prime}, y^{r} \ldots$ are each zero for $c e_{0}(z)$, etc., and $y, y^{\prime \prime}, y^{\text {iv }} \ldots$ are each zero for $s e_{1}(z)$, etc. Hence two determinants are again derived,

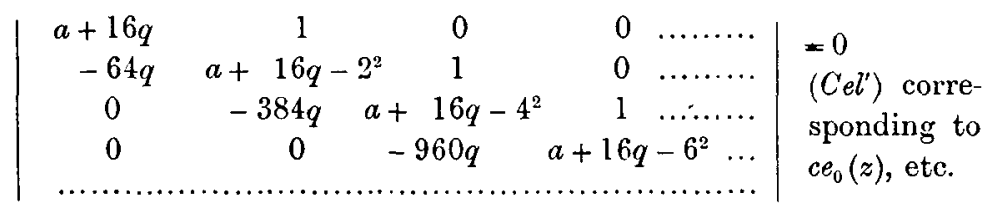

and

$$
\begin{array}{cccc|c}
a+16 q-1^{2} & 1 & 0 & 0 \ldots \ldots \ldots & =0 \\
-192 q & a+16 q-3^{2} & 1 & 0 \ldots \ldots \ldots & \left(S o l^{\prime}\right) \text { corre- } \\
0 & -640 q & a+16 q-5^{2} & 1 \ldots \ldots \ldots & \text { sponding to } \\
0 & 0 & -1344 q & a+16 q-7^{2} \ldots & s e_{1}(z), \text { etc. }
\end{array}
$$

The dash at $\mathrm{Cel}$ and $\mathrm{Sol}$ signifies that $a+16 q$ is the principal term instead of $a-16 q$.

Determinant $C e l^{\prime}$ when truncated produced the same relations as Cel, whereas, 'ol', corresponding to $s_{1}(z)$, gave

$$
a=8 q-8 q^{2}-8 q^{3} \ldots
$$


\$5. Determinant corresponding to $\mathrm{se}_{2}(z), \mathrm{se}_{4}(z)$, etc.

$s e_{2}(z), s e_{4}(z)$, etc., cannot be expressed as polymonials in $\sin z, \cos z, \sin ^{2} z$, or $\cos ^{2} z$, hence none of the foregoing substitutions will give the relations corresponding to these solutions, but, as they can be expressed in terms of $\sin 2 z, \sin 4 z$, etc., if they be divided by $\sin z, \frac{s e_{2}(z)}{\sin z}, \frac{s e_{4}(z)}{\sin z}$, etc., can be expressed in terms of $\cos z$. Accordingly, the substitution $y=\xi \sin z$ was made in Mathieu's equation, and the equation

$$
\left(1-x^{2}\right) \xi^{\prime \prime}-3 x \xi+\left(a-16 q-1^{2}+32 q x^{2}\right) \xi=0
$$

was derived, in which $x=\cos z$. Now, $g e_{1}(z), s e_{3}(z)$, etc., may also be expressed in terms of $\cos z$, when divided by $\sin z$, and hence this equation will give a determinunt corresponding to these solutions as well. The two determinants derived were

\begin{tabular}{|c|c|c|c|c|}
\hline$a-16 q-1$ & 1 & 0 & .......... & $=0$ \\
\hline $64 q$ & $a-16 q-3^{2}$ & 1 & 0 & (Sol) corre- \\
\hline 0 & $384 q$ & $a-16 q-5^{2}$ & 1 & sponding to \\
\hline 0 & 0 & $960 q$ & $a-16 q-7^{2} \ldots$ & $\operatorname{se}_{1}(z), \operatorname{se}_{3}(z)$, \\
\hline
\end{tabular}

and

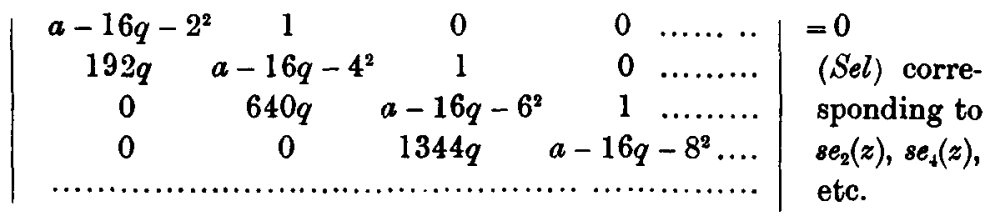

Determinant $S o l$ when truncated produced the same relations as $S o l '$, whereas $S e l$, corresponding to $s e_{2}(z)$, gave

$$
a=-\frac{16}{3} q^{2}+\frac{4}{2} \frac{0}{7} q^{4} \ldots
$$

§6. Notes on the foregoing Determinants.

Determinants $\mathrm{Cel}$ and $\mathrm{Col}$ were got from the equation

$$
\left(1-x^{2}\right) y^{\prime \prime}-x y^{\prime}+\left(a-16 q+32 q x^{2}\right) y=0,
$$

and $\mathrm{Cel}^{\prime}$ and $S o l^{\prime}$ from the equation

$$
\left(1-x^{2}\right) y^{\prime \prime}-x y^{\prime}+\left(a+16 q-32 q x^{2}\right) y=0 .
$$


It will be noticed that these equations difer only in the sign of $q$. As the relations corresponding to $c e_{0}(z), c e_{2}(z)$, etc., were got from both of these equations, these relations must involve only even powers of $q$, so that the interchange of the sign of $q$ may leave them unaltered. This agrees with the values found for $q$,

$$
a=-32 q^{2}+224 q^{4} \ldots \text { etc. }
$$

Hence determinant $C e l=$ determinant $C e l$.

Again, the first of these equations gave determinant $\mathrm{Col}$ corresponding to $c e_{1}(z)$, etc., and the second determinant Sol corresponding to $s e_{1}(z)$, etc. Therefore the relation corresponding to $s e_{1}(z)$ may be got from that corresponding to $c e_{1}(z)$ by changing the sign of $q$. This agrees with the values found,

$$
c e_{1}(z)=-8 q-8 q^{2}+8 q^{3} \ldots \text { and } s e_{1}(z)=+8 q-8 q^{2}-8 q^{3} \ldots
$$

Similarly in $\S 5 y=\xi \cos z$ might have been substituted, and the equation

$$
\left(1-x^{2}\right) \xi^{\prime \prime}-3 x \xi^{\prime}+\left(a+16 q-1^{2}-32 q x^{2}\right) \xi=0
$$

have been got instead of

$$
\left(1-x^{2}\right) \xi^{\prime \prime}-3 x \xi^{\prime}+\left(a+16 q-1^{2}+32 q x^{2}\right) \xi=0,
$$

and determinants corresponding to $c e_{1}(z), c e_{3}(z)$, etc., and $s e_{2}(z)$, $s e_{1}(z)$, etc., derived. These would be

\begin{tabular}{|ccccc|c}
$a+16 q-1^{2}$ & 1 & 0 & 0 & $\ldots \ldots \ldots$ & $=0$ \\
$-64 q$ & $a+16 q-3^{2}$ & 1 & 0 & $\ldots \ldots \ldots$ & $\left(C o l^{\prime}\right)$ corre- \\
0 & $-384 q$ & $a+16 q-5^{2}$ & 1 & $\ldots \ldots \ldots$ & sponding to \\
0 & 0 & $-960 q$ & $a+16 q-7^{2} \ldots$ & $c e_{1}(z)$, etc.
\end{tabular}

and

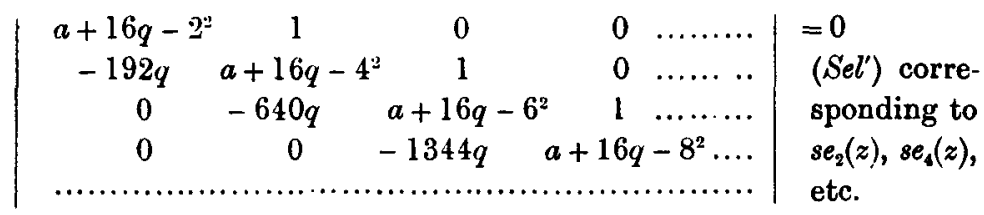

Hence the relation corresponding to $s e_{2}(z), s e_{4}(z)$, etc., must involve only even powers of $q$. This agrees with the relation found, $a=-\frac{16}{2} q^{2}+\frac{4}{2} \frac{0}{7} q^{4} \ldots$. 
It will be noticed that

(1) $\mathrm{Cel}^{\prime}$ and $\mathrm{Col}^{\prime}$ have another diagonal $-64 q,-384 q$, etc. $\mathrm{Sel}^{\prime}$ and $\mathrm{Sol}^{\prime} \quad$ - $\quad$ - $\quad-\quad-192 q,-640 q$, etc.

(2) $\mathrm{Cel}$ and $\mathrm{Sol} \quad-\quad-\quad C_{0} \quad 64 q, 384 q$, etc. Col and Sel - - - - $192 q, 640 q$, etc.

The coefficients of $q$ are $\pm 64(r-1)(2 r-3)$ for one set of determinants, and $\pm 64(r-1)(2 r-1)$ for the other, where $r$ is the number of the row in which the quantity occurs.

\section{§7. Hill's Method.}

Assuming for the solution $y=\sum_{n=-\infty}^{\infty} b_{n} e^{2 n i z}$, and substituting Mathieu's equation becomes

$$
\sum_{n=-\infty}^{\infty}(2 n i)^{2} b_{n} e^{2 n i z}+\left(a+8 q e^{2 n i z}+8 q e^{-2 i z}\right) \sum_{n=-\infty}^{\infty} b_{x} e^{2 n i z}=0 .
$$

Equating to zero the coefficients of $e^{2 n i z}$, the relation (which holds true for all integral values of $n$ )

is obtained.

$$
8 q b_{n-1}+\left[(2 n i)^{2}+a\right] b_{n}+8 q b_{n-1}=0
$$

If in this equation values of $n$ from $-\infty$ to $+\infty$ are inserted, a series of equations is derived, the central ones of which are

$$
\begin{array}{lc}
8 q b_{-3}+\left(a-4^{2}\right) b_{-2}+8 q b_{-1}=0 & (n=-2) \\
8 q b_{-2}+\left(a-2^{2}\right) b_{-1}+8 q b_{0}=0 & (n=-1) \\
8 q b_{-1}+a b_{0}+8 q b_{1}=0 & (n=0) \\
8 q b_{0}+\left(a-2^{2}\right) b_{1}+8 q b_{2}=0 & (n=1) \\
8 q b_{1}+\left(a-4^{2}\right) b_{2}+8 q b_{3}=0 & (n=2)
\end{array}
$$

\begin{tabular}{|c|c|c|c|c|c|}
\hline$\ldots \ldots 8 q$ & $a-4^{2}$ & $8 q$ & 0 & 0 & $0 \ldots \ldots$ \\
\hline ..... 0 & $8 q$ & $a-2^{2}$ & $8 q$ & 0 & $0 \ldots \ldots$ \\
\hline$\ldots \ldots 0$ & 0 & $8 q$ & $a$ & $8 q$ & $0 \ldots \ldots$ \\
\hline$\ldots \ldots 0$ & 0 & 0 & $8 q$ & $a-2^{2}$ & $8 q \ldots$. \\
\hline$\ldots \ldots 00$ & 0 & 0 & 0 & $8 q$ & $a-4^{2} .$. \\
\hline
\end{tabular}

Eliminating the $b$ 's we get the doubly infinite determinant 
This infinite determinant of order $2 r+1$ can be split up into two determinants-

$\left|\begin{array}{lcccc}a & 16 q & 0 & 0 & 0 \ldots \ldots \\ 8 q & a-2^{2} & 8 q & 0 & 0 \ldots \ldots \\ 0 & 8 q & a-4^{2} & 8 q & 0 \ldots \ldots \\ 0 & 0 & 8 q & a-6^{2} & 8 q \ldots \ldots \\ 0 & 0 & 0 & 8 q & a-8^{2} \ldots\end{array}\right|=0($ Ceh $)$

and

\begin{tabular}{|c|c|c|c|c|}
\hline$a-2^{2}$ & $8 q$ & 0 & $0 \ldots \ldots$ & \\
\hline $8 q$ & $a-4^{2}$ & $8 q$ & $0 \ldots \ldots$ & \\
\hline 0 & $8 q$ & $a-6^{2}$ & $8 q \ldots \ldots$ & $=0 \quad(\operatorname{Seh})$ \\
\hline 0 & 0 & $8 q$ & $a-8^{2} \ldots \ldots$ & \\
\hline
\end{tabular}

Assuming the expansion for $c e_{0}(z)$ and retaining terms up to $q^{4}$, $c e_{0}(z)=1+\left(4 q-28 q^{3} \ldots\right) \cos 2 z+\left(2 q^{2}-1,00 q^{4} \ldots\right) \cos 4 z+\ldots$

$$
\begin{aligned}
=b_{0} & +b_{1} e^{2 i z} & +b_{2} e^{+i z}+\ldots \\
+b_{-1} e^{-2 i z} & & +b_{-2} e^{-4 i z}+\ldots
\end{aligned}
$$

we see that $b_{1}=b_{-1}, b_{2}=b_{-2}$, etc.

Returning to the equations, substitute these, and the equation for $n=0$ becomes $a b_{0}+16 q b_{1}=0$, while the others become identical in pairs, i.e. equation for $n=r$ is the same as that for $n=-r$. If the $b$ 's be eliminated in the equations, determinant C'eh is derived. If $C e h$ be truncated to $t$ columns and rows,

$$
a=-32 q^{2}+224 q^{4} \ldots \text { corresponding to } c e_{0}(z),
$$

the same relation as was derived from $C e l$.

The leading diagonal, when $q=0$, grives $a=2^{2}, 4^{2}, 6^{2}, \ldots$ and hence the values of $a$ corresponding to $c e_{2}(z), c e_{4}(z)$, may be derived.

$$
\begin{array}{rlrl}
s e_{2}(z)= & \sin 2 z & +\left(\frac{2}{3} q-\frac{5}{3} q^{3}\right) \sin 4 z+\frac{1}{6} q^{2} \sin 6 z+\ldots \\
= & b_{0}+b_{1} e^{2 i z} & +b_{2} e^{4 i z} & +b_{3} e^{6 i z}+\ldots \\
& +b_{-1} e^{-2 i z}+b_{-2} e^{-4 i z} & +b_{-3} e^{-6 i z}+\ldots
\end{array}
$$

Hence $b_{0}=0, b_{1}=-b_{-1}, b_{2}=-b_{-2,2}$ etc.

Substituting in the equations and eliminating the $b$ 's, we derive determinant Seh. As the leading diagonal is $a-2^{2}, a-4^{2}$, etc., 
this determinant will give the values of $a$ corresponding to $s e_{2}(z), s e_{4}(z)$, etc. If it be truncated to 4 columns and rows, and $a+2^{2}$ substituted for $a, a=-\frac{16}{3} q^{2}+\frac{40}{27} q^{4} \ldots$, the value derived from $S e l$.

\$8. Determinants corresponding to $c e_{1}(z), c e_{3}(z)$, etc., $s e_{1}(z)$, $s e_{3}(z)$, etc.

Assuming for the solution $y=\sum_{n=-\infty}^{n=\infty} b_{n} e^{(2 n-1) i z}$, and substituting in Mathieu's equation, we find it becomes

$$
\sum_{n=-\infty}^{\infty}\{(2 n-1) i\}^{2} b_{n} e^{(2 n-1) i z}+\left(a+8 q e^{2 i z}+8 q e^{-2 i z}\right) \sum_{n=-\infty}^{\infty} b_{n} e^{(2 n-1) i z}=0 .
$$

If the coefficients of $e^{(2 n-1) i z}$ be equated to zero, the relation (which holds true for all integral values of $n$ )

$$
8 q b_{n-1}+\left[\{(2 n-1) i\}^{2}+a\right] b_{n}+8 q b_{n+1}=0
$$

is obtained.

In this equation values of $n$ from $n=-\infty$ to $+\infty$ are inserted, and a series of equations is obtained, the central ones of which are

$$
\begin{array}{lc}
8 q b_{-3}+\left(a-5^{2}\right) b_{-2}+8 q b_{-1}=0 & (n=-2) \\
8 q b_{-2}+\left(a-3^{2}\right) b_{-1}+8 q b_{0}=0 & (n=-1) \\
8 q b_{-1}+\left(a-1^{2}\right) b_{0}+8 q b_{1}=0 & (n=0) \\
8 q b_{0}+\left(a-1^{2}\right) b_{1}+8 q b_{2}=0 & (n=1) \\
8 q b_{1}+\left(a-3^{2}\right) b_{2}+8 q b_{3}=0 & (n=2)
\end{array}
$$

If the $b$ 's be eliminated the doubly infinite determinant of order $2 r$ is derived.

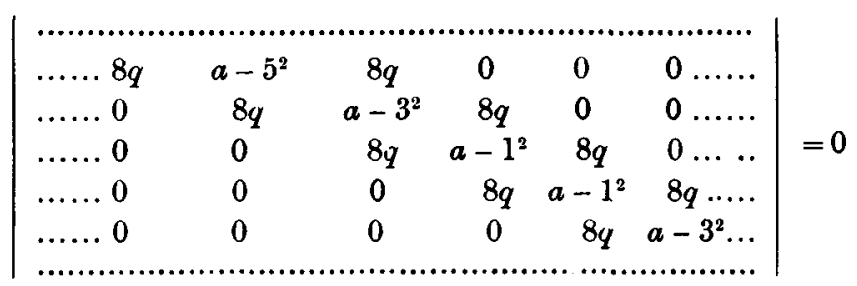


This can be broken up into two determinants

\begin{tabular}{|c|c|c|c|}
\hline$a-1^{2}+8 q$ & $8 q$ & 0 & $0 \ldots$. \\
\hline $8 q$ & $a-3^{2}$ & $8 q$ & $0 \ldots \ldots$ \\
\hline 0 & $8 q$ & $a-5^{2}$ & $8 q \ldots \ldots$ \\
\hline 0 & 0 & $8 q$ & $a-7^{2} \ldots$ \\
\hline
\end{tabular}

and

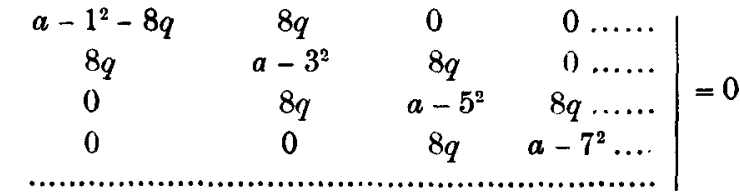

$$
\begin{aligned}
& c e_{1}(z)=\cos z \quad+\left(q-q^{2}\right) \cos 3 z+\ldots \\
& =b_{1} e^{i z} \quad+h_{2} e^{3 i z}+\ldots \\
& +b_{0} e^{-i z}+b_{-1} e^{-3 i z}+\ldots
\end{aligned}
$$

Hence $b_{0}=b_{1}, b_{2}=b_{-1}, b_{3}=b_{-2}$, etc.

Substituting in the equations and eliminating the $b$ 's, we derive determinant Coh. Hence this determinant gives the relation between $a$ and $q$ corresponding to $c e_{1}(z), c e_{3}(z)$, etc. When truncated to 3 columns and rows, $a=-8 q-8 q^{2}+8 q^{3} \ldots$ for $c e_{1}(z)$, the value found from $\mathrm{Col}$.

$$
\begin{aligned}
8 e_{1}(z) & =\sin z & & +\left(q+q^{2}\right) \sin 3 z+\ldots \\
& =b_{1} e^{i z} & & +b_{2} e^{3 i z}+\ldots \\
& +b_{0} e^{-i z} & & +b_{-1} e^{-3 i z}+\ldots
\end{aligned}
$$

Hence $b_{0}=-b_{1}, b_{-1}=-b_{2}, b_{-2}=-b_{3}$, etc.

If these substitutions be made in the equations and the $b$ 's eliminated, determinant $S o h$ is got. Accordingly this determinant gives the relation between $a$ and $q$ corresponding to $s e_{1}(z), s e_{3}(z)$, etc. When truncated to 3 columns and rows, $a=8 q-8 q^{2}-8 q^{3} \ldots$ for $s e_{1}(z)$, the value found from Sol.

If it be remembered that instead of $8 q$ and $8 q$ in the two diagonals 1 and $64 q^{2}$ may be substituted, it will be seen that a change of sign in $q$ will leave the determinants corresponding to $c e_{0}(z)$ and $s e_{2}(z)$ unaltered, whereas it will convert the determinant corresponding to $c e_{1}(z)$ into that of $s e_{1}(z)$ and vice versa. 


\section{4}

It may be remarked that in order to get the value of $a$ correct to $q^{n}, n+1$ rows and columns had to be taken in the determinants derived by the first method, whereas by Hill's method $n$ rows and columns were sufficient, and that the calculations were far easier.

\section{\$9. Equivalence of the Equations and Determinants.}

In the first method $c e_{0}(z)$ was taken as equal to

$$
A+B \cos ^{2} z+C \cos ^{4} z \ldots
$$

and in the second method equal to

$$
a+b \cos 2 z+c \cos 4 z \ldots
$$

We can therefore, by considering such equations as

$$
\cos 2 z=2 \cos ^{2} z-1, \cos 4 z=8 \cos ^{4} z-8 \cos ^{2} z+1 \text {, etc. }
$$

get a series of relations connecting $A, B, C, \ldots$ with $a, b, c, \ldots$. Call these relations $I$. Now in the first method we get linear equations for $A, B, C, \ldots$ Call these equations $I I$. In the second method we get linear equations for $a, b, c, \ldots$. Let these be called $I I I$. Equations $I I I$. must be transformable into equations $I I$. by means of relations $I$., and hence the determinants derived from these equations equivalent.

As an example take $C e l$ and $C e h$.

$$
\begin{aligned}
& y=A+B \cos ^{2} z+C \cos ^{4} z+D \cos ^{6} z+\ldots \\
& \text { or } y=A+B x^{2}+C x^{4}+D x^{6}+\ldots \quad \text { where } x=\cos z \text {. } \\
& \therefore y=A, y^{\prime}=0, y^{\prime \prime}=2 B, y^{\prime \prime \prime}=0, y^{\mathrm{iv}}=24 C, y^{\mathrm{v}}=0, y^{\mathrm{v} 1}=720 \mathrm{D} \text {, } \\
& \text { when } x=0 \text {. } \\
& \text { Again, } y=b_{0}+2 b_{1} \cos 2 z+2 b_{2} \cos 4 z+2 b_{3} \cos 6 z \ldots \\
& =b_{0}+2 b_{1}\left(2 \cos ^{2} z-1\right)+2 b_{2}\left(8 \cos ^{4} z-8 \cos ^{2} z+1\right) \\
& +2 b_{3}\left(32 \cos ^{6} z-48 \cos ^{4} z+18 \cos ^{2} z-1\right)+\ldots \\
& \therefore y=b_{0}-2 b_{1}+2 b_{2}-2 b_{3}+\ldots \\
& y^{\prime \prime}=8 b_{1}-32 b_{2}+72 b_{3}-\ldots \\
& y^{\text {iv }}=384 b_{2}-2304 b_{3}+\ldots \quad \text { etc. }
\end{aligned}
$$

The equations from which $C e h$ was derived were:-

$$
\begin{gathered}
a b_{0}+16 q b_{1}=0, \quad 8 q b_{0}+(a-4) b_{1}+8 q b_{2}=0, \\
8 q b_{1}+(a-16) b_{2}+8 q b_{3}=0, \quad \text { etc. }
\end{gathered}
$$


To derive the first equation from which $\mathrm{Cel}$ was got, multiply the first of these equations by 1 , the second by -2 , the third by +2 , etc., $i e$. by the coefficients of $b_{0}, b_{1}, b_{2}, \ldots$ in the relation connecting them with $y$. Add these and equate to zero.

$$
\begin{aligned}
\therefore \quad & \left(a b_{0}+16 q b_{1}\right)-2\left[8 q b_{0}+(a-4) b_{1}+8 q b_{2}\right] \\
& 2\left[8 q b_{1}+(a-16) b_{2}+8 q b_{3}\right]+\ldots=0 .
\end{aligned}
$$

Rearranging this equation we have

$$
(a-16 q)\left(b_{0}-2 b_{1}+2 b_{2}-\ldots\right)+\left(8 b_{1}-32 b_{2}+72 b_{3}-\ldots\right)=0,
$$

i.e. $(a-16 q) y+y^{\prime \prime}=0$, the first of the equations from which $\mathrm{Cel}$ was got.

Again, multiplying the second of the equations by 8 , the third by -32 , the fourth by $+72, \ldots$ etc., i.e. by the coefficients of $b_{1}, b_{2}, b_{3}, \ldots$ in the relation connecting them and $y^{\prime \prime}$, and adding and equating to zero, we have

$$
\begin{gathered}
8\left[8 q b_{0}+(a-4) b_{1}+8 q b_{2}\right]-32\left[8 q b_{1}+(a-16) b_{2}+8 q b_{3}\right] \\
+72\left[8 q b_{2}+(a-36) b_{3}+8 q b_{4}\right]-\ldots=0 .
\end{gathered}
$$

Rearranging this equation, it becomes

$64 q\left(b_{0}-2 b_{1}+2 b_{2}-2 b_{3} \ldots\right)+(a-16 q-4)\left(8 b_{1}-32 b_{2}+72 b_{3} \ldots\right)=0$, i.e. $64 q y+(a-16 q-4) y^{\prime \prime}=0$, the second of the equations from which $C e l$ was got.

Continuing in an exactly similar manner we can derive the other equations from which $C^{\prime} e l$ was got.

Hence the determinant $\mathrm{Cel}$ is equivalent to $\mathrm{Ceh}$.

It will be noticed that the multipliers of the equations

$$
a b_{0}+16 q b_{1}=0 \text {, etc., }
$$

were the coefficients of $b_{0}, b_{1}$, etc., in the relations connecting them and $y, y^{\prime \prime}$, etc., and that these coefficients were the coefficients of $\cos ^{r} z$ in the expansion of $\cos 2 n z$ in terms of $\cos z$, multiplied by certain factors.

Similarly it can be shown that

$$
C e l^{\prime}=C e h, C o l=C o l^{\prime}=C o h, S e l=S e l^{\prime}=S e h, S o l=S o l^{\prime}=S o h \text {. }
$$

The following table shows the various multipliers to convert the equations of the second method into the first two equations of the first method. 


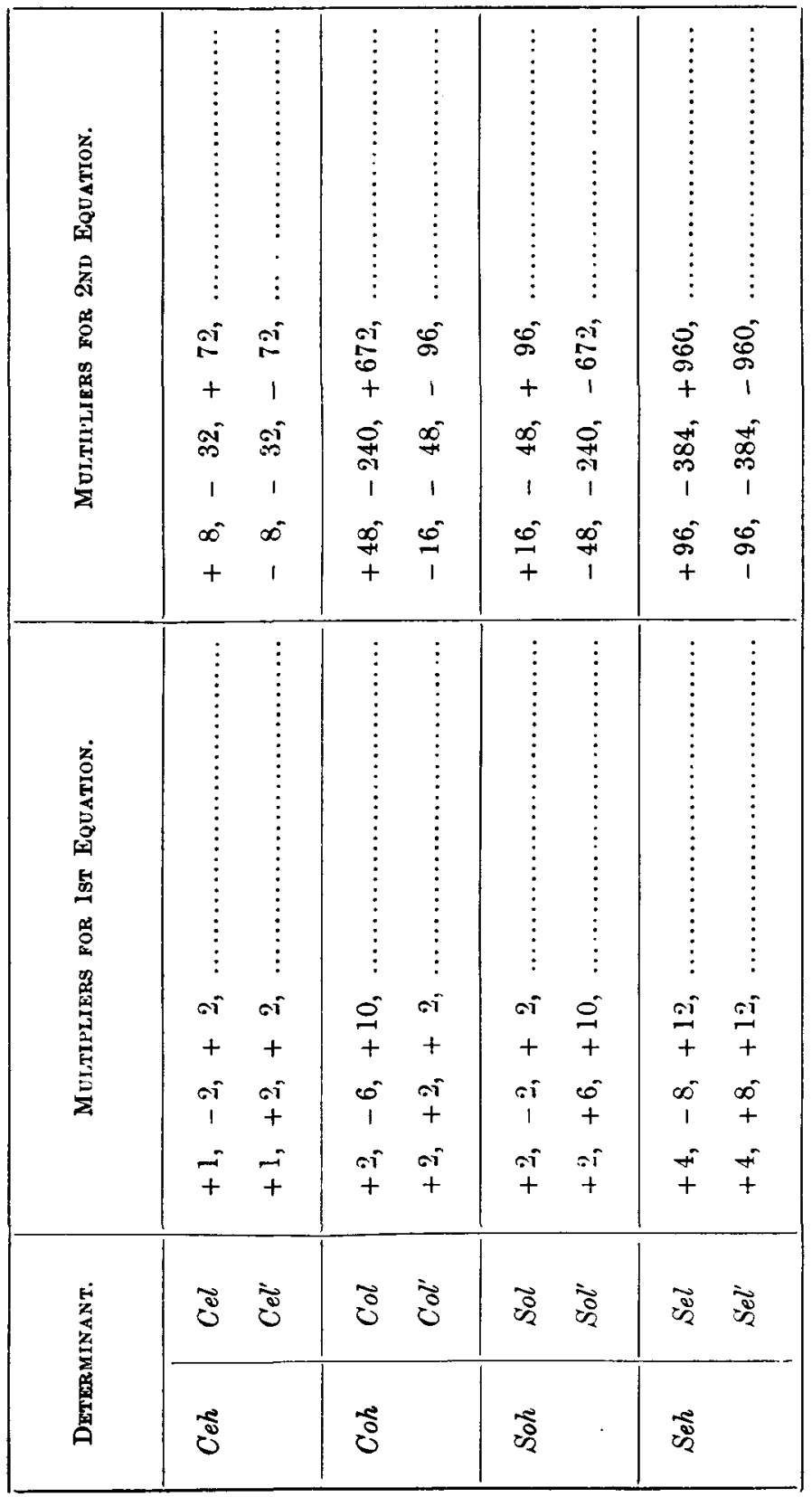




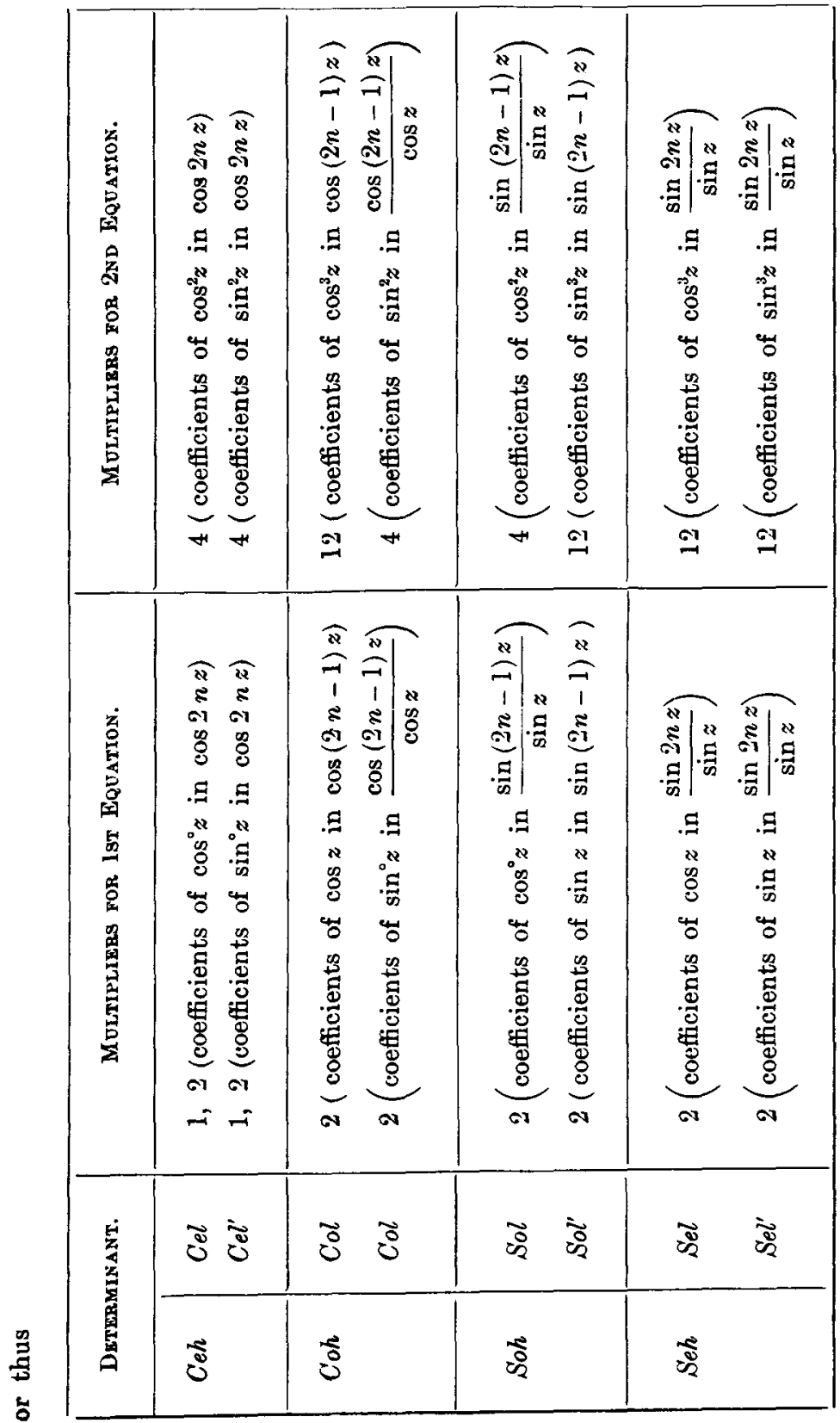

10 Vol. 33 
When it is remembered that changing the sign of $q$ converts $\mathrm{Cel}$ into $\mathrm{Cel}^{\prime}$, and Sel into Sel', but Col into Sol' and Col' into Sol a glance at the above tables shows that they possess, in addition to others, the same interesting features. It will be noted, for example, $\mathrm{Cel}$ and $\mathrm{Cel}^{\prime}$ have the same multipliers, differing only in the sign of every second multiplier, and from the second table that the alteration is merely the substitution of sine for cosine. The same applies to $S e l$ and $\mathrm{Sel}^{\prime}$. But in the case of the odd functions, $\mathrm{Col}$ and $S o l^{\prime}$ correspond in this way, and $\mathrm{Col}^{\prime}$ and Sol. A reference to the transformation of $\mathrm{Ceh}$ into $\mathrm{Cel}$, and the corresponding one of $\mathrm{Ceh}$ into $\mathrm{Cel}^{\prime}$ at once supplies the reason.

In conclusion, I desire to thank Professor Whittaker, at whose suggestion this investigation was begun, for much useful advice during its progress. 